\title{
DECISION SUPPORT SYSTEM FOR PLANNING \\ TELECOMMUNICATION NETWORKS: A CASE STUDY APPLIED TO THE ANDALUSIAN REGION
}

P Cortes, L Onieva, J Larrañeta and JM Garcia

University of Seville, Spain

Network planning is essential to design real Broadband Integrated Services Digital Networks (B-ISDN). This paper presents an OR application to the design of an optic fibre network for the andalusian region. The economical appraisal is the main consideration in order to take the appropriate decisions: hub location, region sizes and selection of the urban nodes that will receive telecommunication contents. A Decision Support System with a graphic interface that allows interactive analysis of different scenarios is presented. The system contains a set of mathematical programming models and it has the capability to dynamically construct and solve instances of those models. In addition, it provides data preparation and reports. The system is an integrated, user-friendly and powerful tool to make planning studies by firms developing cable network systems in the telecommunications market.

Keywords: B-ISDN, Decision Support System, case study, optimization. 


\section{Introduction}

This paper presents a Decision Support System (DSS), developed as integrated, userfriendly and powerful software that can help the designer in the telecommunication network planning process. Sprague and Carlson ${ }^{1}$ defined DSS as interactive computerbased systems that help decision-makers utilise data and models to solve unstructured problems. Wynne ${ }^{2}$ shows how DSS must help OR acting as supportive tools under the management user's control, which do not attempt to automate the entire process, predefining objectives or imposing solutions. Following these lines our tool lets the user include in the decision process more information than usually allowed with other approaches, providing data preparation and reports, as well as, the ability to deal with less structured situations and the flexibility to evaluate alternative scenarios.

The use of DSS constitutes an important tool for firms developing network communication systems in the telecommunication market. In this same line, Cosares et $\mathrm{al}^{3}$ proposed a DSS for designing interconnected SONET (the US transmission standard) rings. More recently and related to this research, Medova ${ }^{4}$ has presented a visualisation tool for B-ISDN backbones planning.

Our DSS has been applied in the optic fibre telecommunication network planning for the Andalusian region (the south most region of Spain). The Andalusian Regional Government divided this geographical area in four official demarcations after the 
technical $^{5}$ study made by the Engineering School of Seville. The application we will describe in this paper can be used for each of the demarcations as well as for the whole region.

Figure 1 depicts the five territorial regions to be covered by the network:

\$ Demarcation I (including the Granada, Jaen and Almeria provinces)

\$ Demarcation II (including the Cordoba and Malaga provinces)

\$ Demarcation III (including the Seville province)

\$ Demarcation IV (including the Huelva and Cadiz provinces)

\$ Andalusia (including the four demarcations)

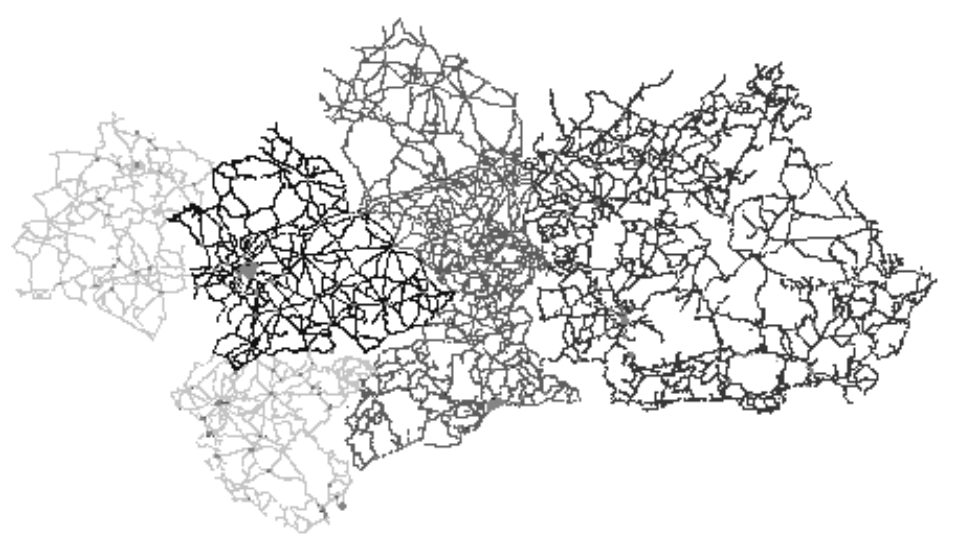

Figure 1 Geographic framework

The rest of the paper deals with the presentation of a DSS to design telecommunication networks. Firstly a section introducing the problem is presented. 
Secondly the Decision Support System is introduced indicating its main structure as well as the mathematical models included. Subsequently the graphic interface of the application and several figures showing the case study results are detailed and discussed. Finally, we review the main aspects in the conclusions section.

\section{Problem description}

The problem arises when a cable telecommunication network has to be set up across a set of municipalities and different associated decisions have to be taken in account.

In this context, the road and the railway networks represent the physical support to lay the optic fibre cables shaping the arcs of the graph. When a cable network has to be set up, the main component of the cost function becomes the construction of the ditches and the conduits (representing up to eighty per cent of the total cost). Also the optic fibre, the transmitter, receiver and regenerator costs are associated to the arc length. So, the length of the arc can be used as an approximation of all these costs.

The main goal is to obtain a set of arcs supporting the telematic network infrastructure and linking an established set of municipalities at minimum cost.

Here some strategic concepts appear as key decisions. One of these concepts is the coverage of the network. Network coverage can be defined according to the 
municipalities receiving telematic services, and can be indexed or stated using different criteria. In this line, the designer can incorporate criteria based on population (size of the city), income (average purchasing power of the city), or any other combination of them, as well as other relevant criteria related to the political or strategic decisions of the firm (for example the introduction of some specific municipalities). The criterion selection will affect the overall goal of the model and will reflect the preference of all actors in the decision problem. So, the level of coverage works as an established constraint for the problem.

All this social-economical background data has been provided by the Statistical Institute of Andalusia (IEA) through its Municipal Information System of Andalusia $\left(\operatorname{SIMA}^{6}\right)$.

\section{The Decision Support System}

Decision Support Systems appear as necessary and integrated tools for all the planning processes. Telecommunication network planning involves a large number of variables and constraints. This DSS allows the user to test alternative scenarios, select alternative decisions, examine the effectiveness of each of such solution, save those solutions of interest and develop multi-stage processes. We present the DSS in the way defined by Connell and Powell ${ }^{7}$, i.e., integrating a whole with the designer.

The DSS is generated through the integration of the social-economical data provided 
by the IEA into the geographical data supplied by the Cartography Institute of Andalusia (ICA) in ARCVINFO format. The data processing provides an abstracted graph representing the whole of the andalusian municipalities and the possibilities of connection among them.

\section{The structure}

The DSS is conceived as an intermediate stage between the data sources (topological and social-economical data) and the final solution, i.e., the telecommunication network. However, the designer must take the critical decisions relative to the network coverage as well as the investment budgets. Figure 2 describes the application structure.
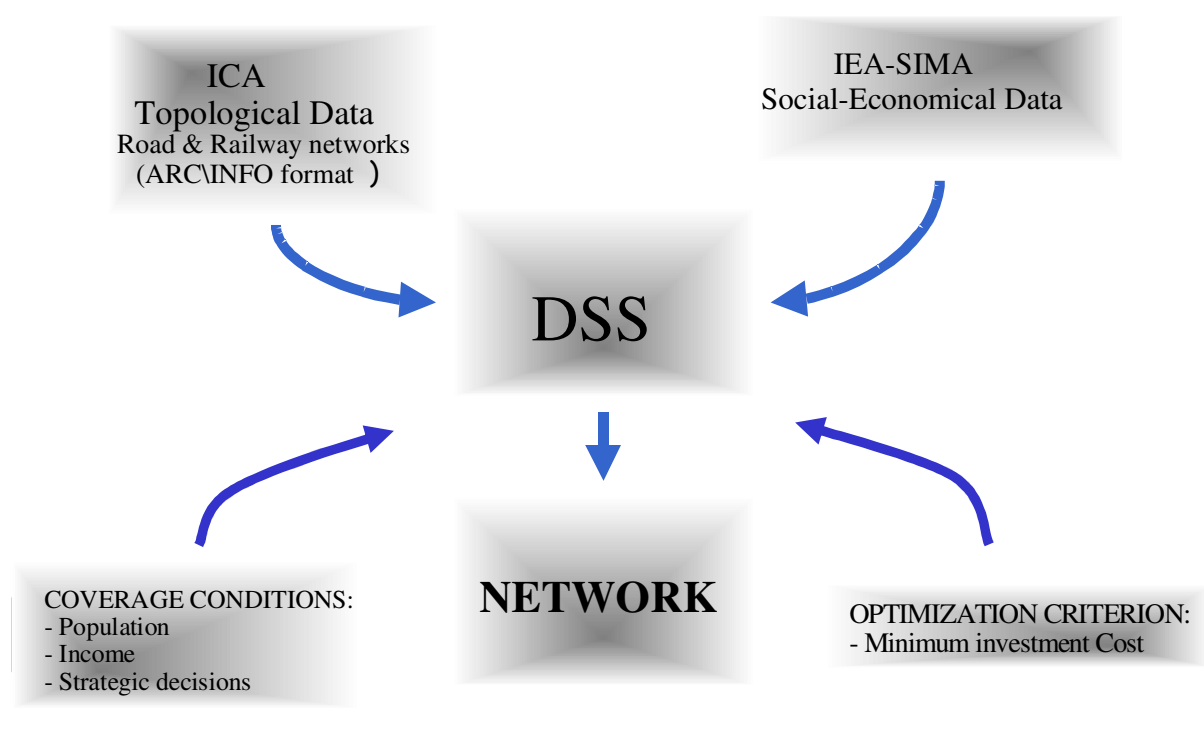

Figure 2 DSS' structure

Initially, the network abstraction from the ICA provides an underlying large graph.

Table 1 shows the graph size for each demarcation. The table includes all the roads 
(freeways, highways, national and regional roads) as well as the railway network.

Table 1 Underlying graph in each demarcation

\begin{tabular}{lccccc}
\hline $\begin{array}{l}\text { Demarcation } \\
\text { GR-JA-AL }\end{array}$ & CO-MA & SE & CA-HU & Andalusia \\
& $($ A-I $)$ & $($ A-II $)$ & (A-III) & (A-IV) & \\
\hline $\begin{array}{l}\text { Number of nodes } \\
\text { Number of arcs }\end{array}$ & 1,094 & 843 & 412 & 649 & 4,773 \\
Number of & 1,507 & 1,246 & 619 & 916 & 4,059 \\
municipalities & 300 & 159 & 100 & 118 & 677 \\
\hline
\end{tabular}

Secondly, SIMA provides the social and economical data for each municipality in the region. The designer takes the appropriate decisions to establish the nodes that should be covered. These will become the terminal nodes of the network. The tool allows the user to select these terminals using strategic or parametric criteria. The DSS presents two kind of selecting parameters:

\$ Population index.

\$ Income index.

The first one indicates the size of the municipality. The second one reflects the purchasing power in the municipality, which is calculated as the total incomes related to the total housings in the city. It is an index related to the family income that is the usual measure unit in the telecommunication services market.

The mathematical model

There exist diverse models to state the topological design of telecommunication 
networks. Most of them are based on multicommodity flow formulations, along with solution methods to reach good feasible solutions. Chang and Gavish ${ }^{8}$ presented different formulations and algorithms depending on the size of the problem. Tcha and Yoon $^{9}$ and later Yoon et $\mathrm{al}^{10}$ developed specific formulations for the design of hierarchical networks. Cortes ${ }^{11}$ has shown that tree oriented formulations can represent quite accurately situations in which basic infrastructure, including ditches and conduits, has to be implemented. Lee et $\mathrm{al}^{12}$, Hall ${ }^{13}$ and Gouveia ${ }^{14}$ have developed topologies based on the capacitated minimum spanning tree (CMST). The difficulty and time delays associated to obtain good feasible solutions make it difficult to integrate these approaches when different alternatives have to be tested.

The topological shape of the basic infrastructure supporting the cable telecommunication network has to establish the links joining a set of nodes having coverage. Thus, there exist a set of nodes to be linked (municipalities with coverage) and another set of nodes without coverage (this set consists of the rest of municipalities without coverage and the physical intersections of the network links). This problem is stated as the Steiner problem in networks that Winter ${ }^{15}$ summarised as:

\$ Given: $\quad$ A non directed graph $G=(N, A)$ with $n$ nodes and $m$ arcs with costs $c_{j}$, and a subset $Z \subseteq N$ with $p$ nodes called terminals. The rest of the nodes are called Steiner nodes.

\$ Find: $\quad$ A network $G_{z} \subseteq G$ joining all the terminal nodes in $Z$ at minimum cost. 
This network can include some of the Steiner nodes but has not to include all the Steiner nodes.

In our context, terminals represent the municipalities with coverage and Steiner nodes represent the rest of municipalities without coverage and the physical intersections of the network. So, the network $G_{z}$ will link all the municipalities receiving coverage while the arcs will represent the facilities where conduits and cables will be placed.

Hence, the Steiner problem formulation in networks, following Aneja ${ }^{16}$, is to minimise:

$$
\sum_{j=1}^{m} c_{j} x_{j}
$$

subject to:

$$
\begin{array}{ll}
\sum_{j=1}^{m} a_{i j} x_{j} \geq 1 & \forall i=1,2, \ldots, q \\
x_{j}=0,1 & \forall j=1,2, \ldots, m
\end{array}
$$

where:

$x_{j}=1$ if the $\operatorname{arc} j$ belongs to the Steiner network

$x_{j}=0$ if the $\operatorname{arc} j$ does not belong to the Steiner network

$m=$ number of arcs in the graph

$q=$ number of the total cut-sets for all possible partitions leaving at least one terminal node in each partition. Id est, $\left\{W, W^{\prime}\right\}$ is a partition of $N$ such that $Z \cap W \neq \varnothing$ and $Z$ $\cap W^{\prime} \neq \varnothing$, so $C_{l}, \ldots, C_{q}$ are all the feasible partitions.

$a_{i j}=$ parameter equal to 1 if the $\operatorname{arc} j$ belongs to the cut $C_{i}, 0$ otherwise. 
The constraint establishes that every terminal node has to receive coverage, so there will be at least one arc joining it to the network. The objective function is to minimise the sum of the costs of arcs in the Steiner network.

\section{Graphic interface and results}

The DSS lets the user dynamically interact with different scenarios. Scenarios can be selected using population criteria, income criteria, or any other direct selection introduced by the designer by clicking into the municipality pixel. The tool also allows the user to analyse evolutionary investment scenarios.

A library of solutions can be constructed for subsequent access and modification. These solutions include interactive access to the internal data associated to each solution. The software constructs automatic technical reports about the analysed scenarios. Figure 3 depicts the tool graphic interface for the case of the Demarcation I that includes the Granada, Jaen and Almeria provinces. 


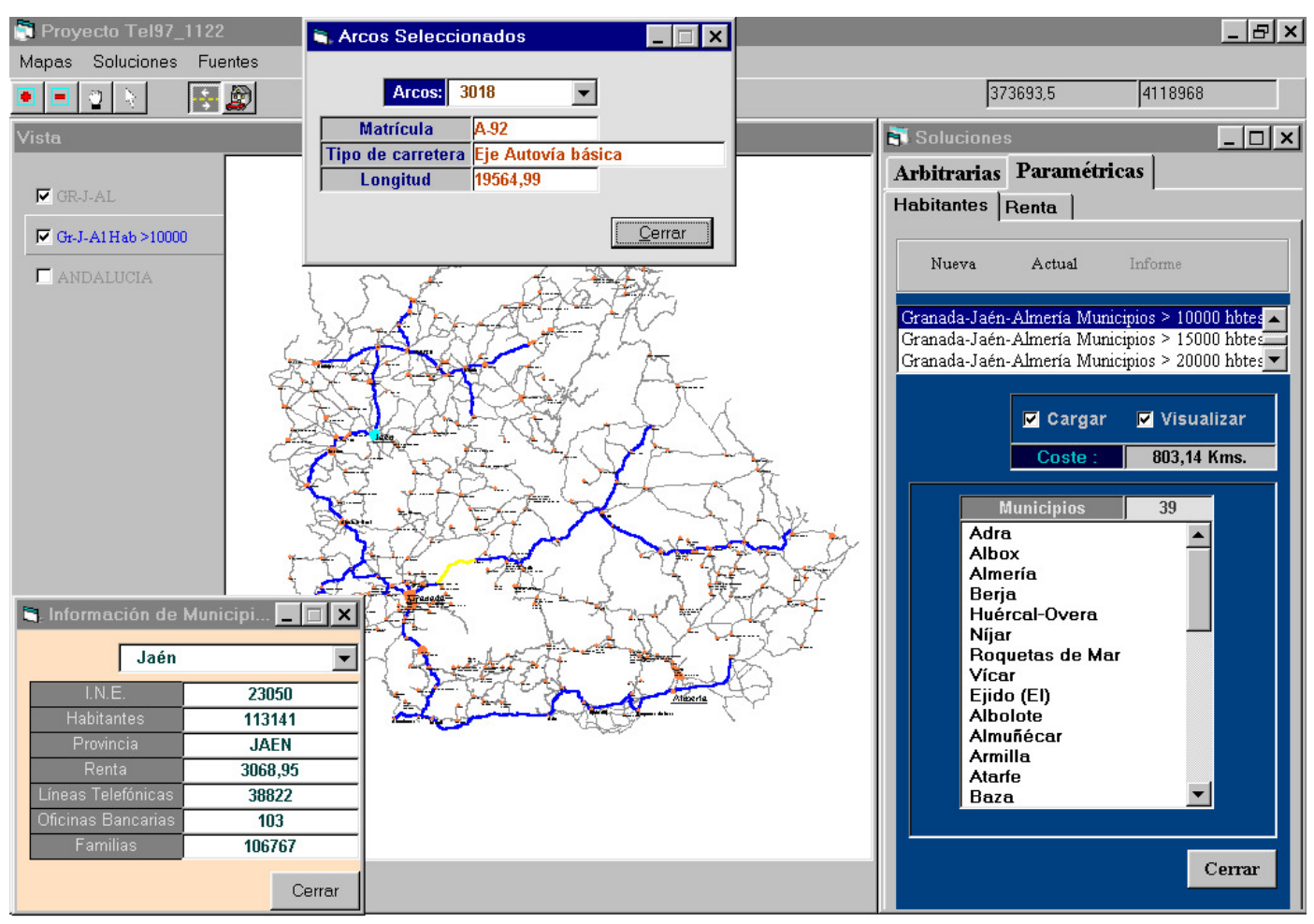

Figure 3 DSS' graphic interface

The central window shows the underlying map and the overlapped telematic network.

The right window shows the different selection criteria, the municipalities receiving coverage for the selected criteria and the total cost. The window on the left reflects the selected scenarios being analysed. There are additional windows showing the social and economical data from the selected municipality, obtained by clicking on it in the graphic display. There are also windows reflecting the characteristics of the selected arcs. 


\section{Solutions of the case study}

Diverse scenarios are shown next. All of them have been selected using the population or income selection criteria as the coverage constraint. The upper cells of the display show the demarcation area, the criteria to select the network coverage and the resulting network length in kilometres. The lower cells of the display show the graphic representation of the geographical area with the final telematic network and the zoom in the main municipalities in the area.

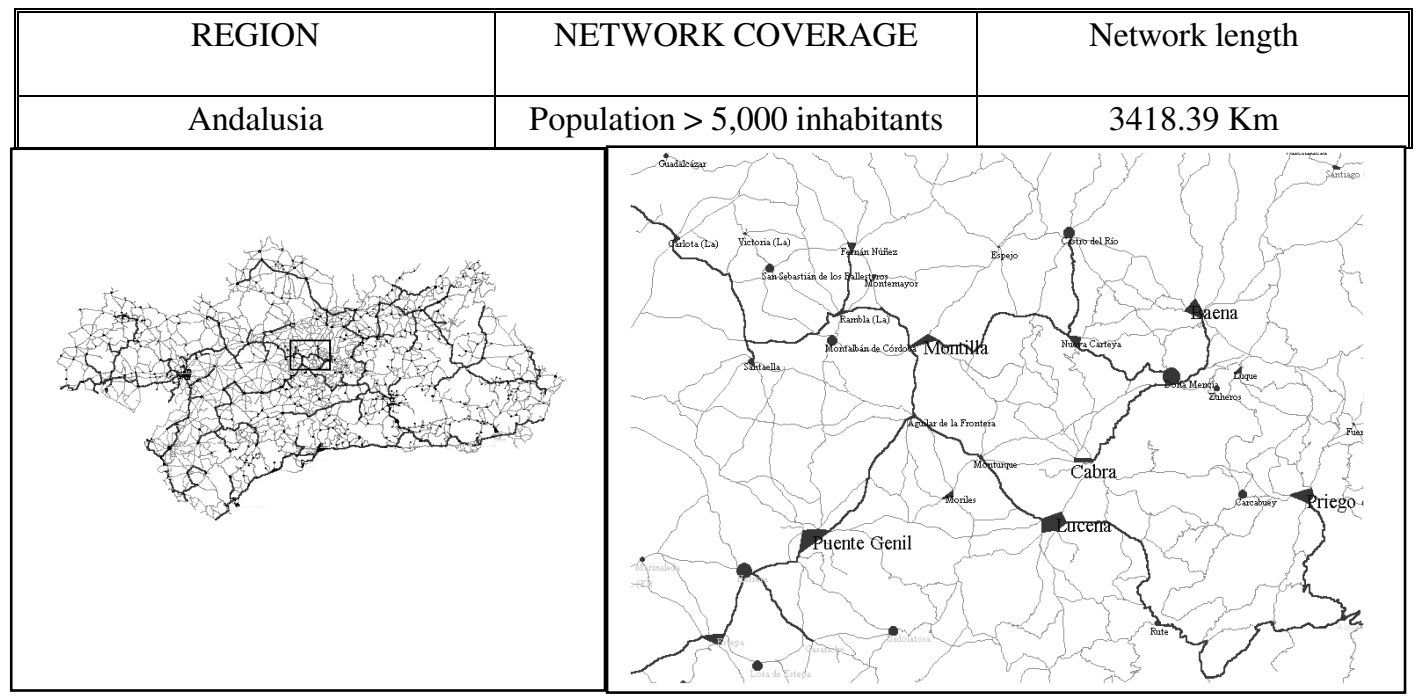

Figure 4 Andalusia

This first solution (figure 4) shows a large network over most of the towns in the region (a coverage equivalent to municipalities larger than 5,000 inhabitants). This was a special request from the Regional Government of Andalusia. The Government 
considered of highest priority to construct a large coverage network extended to the most part of the andalusian population, so the firms developing cable services had to offer these conditions in their proposals to the public auction.

\begin{tabular}{|c|c|c|c|}
\hline \multicolumn{1}{|c|}{ REGION } & NETWORK COVERAGE & Network length \\
\hline Dem. I Granada-Jaen-Almeria & \multicolumn{2}{|c|}{ Population $>10,000$ inhabitants } & $803.14 \mathrm{Km}$ \\
\hline & &
\end{tabular}

Figure 5 Demarcation I

The display of figure 5 reflects a possible situation for the Demarcation I. This Demarcation has the largest area in the region as well as the most disseminated population. In this scenario, firms have to make a very important investment to connect all the municipalities. The investment process would give first priority connecting the main municipalities in order to advance the returns. The rest of the towns in the region would be incorporated in a second stage. 


\begin{tabular}{||c|c|c|}
\hline REGION & NETWORK COVERAGE & Network length \\
\hline Dem. II Malaga-Cordoba & Population $>15,000$ inhabitants & $570.26 \mathrm{Km}$ \\
\hline & &
\end{tabular}

Figure 6 Demarcation II

This situation depicts a scenario where the main goal is to achieve the maximum profit while an important coverage is attained. Municipalities with more than 15,000 inhabitants are included under the assumption of a high correlation between the size of the community, the appreciation of cable services by the population and the location of the main business firms.

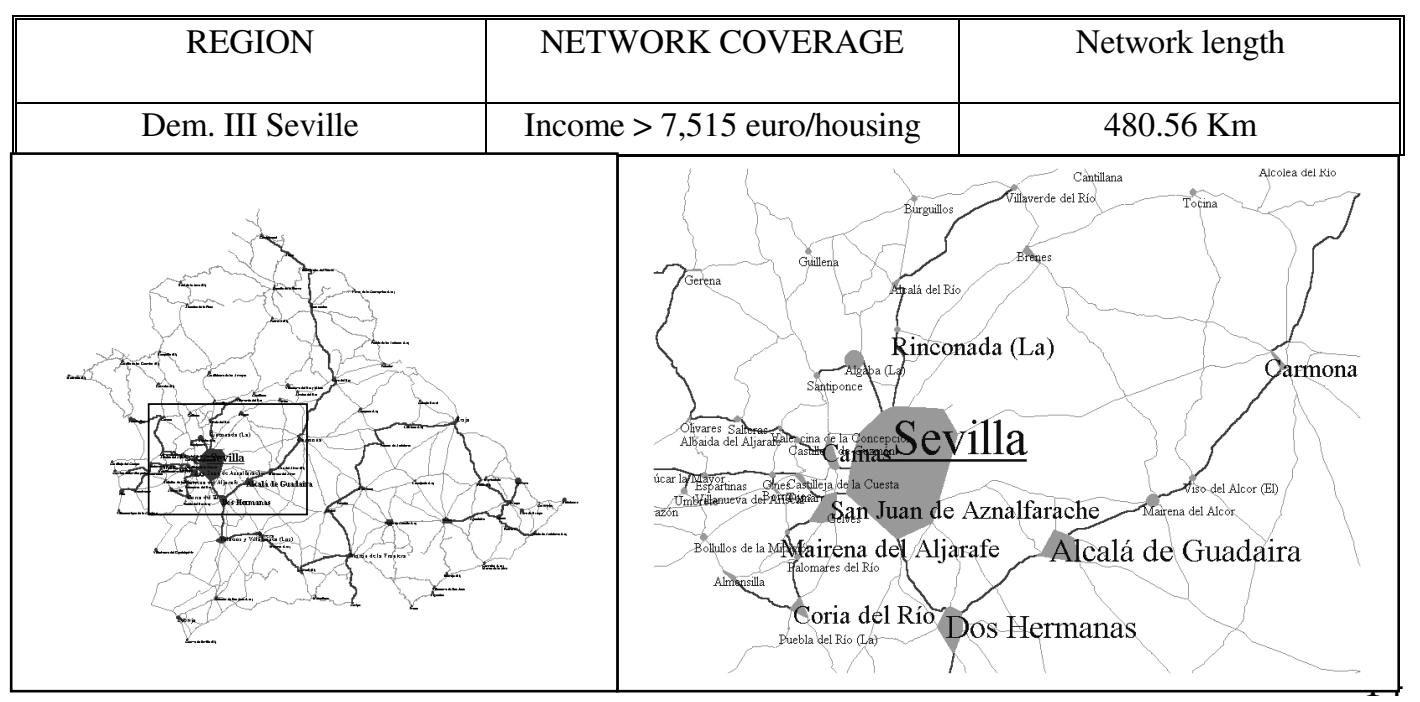

Figure 7 Demarcation III 
The case showed for Demarcation III reveals a situation in which the main criteria in the network performance is the purchasing power related to the total housings of the municipality. Plausibly most of the firms active in the telecommunication market are interested in the pay back and profit generation, so under this situation income criteria turns to be the most attractive. Figure depicts such a case for the Seville province Demarcation. This scenario includes the city of Seville and the larger surrounding towns of its metropolitan area.

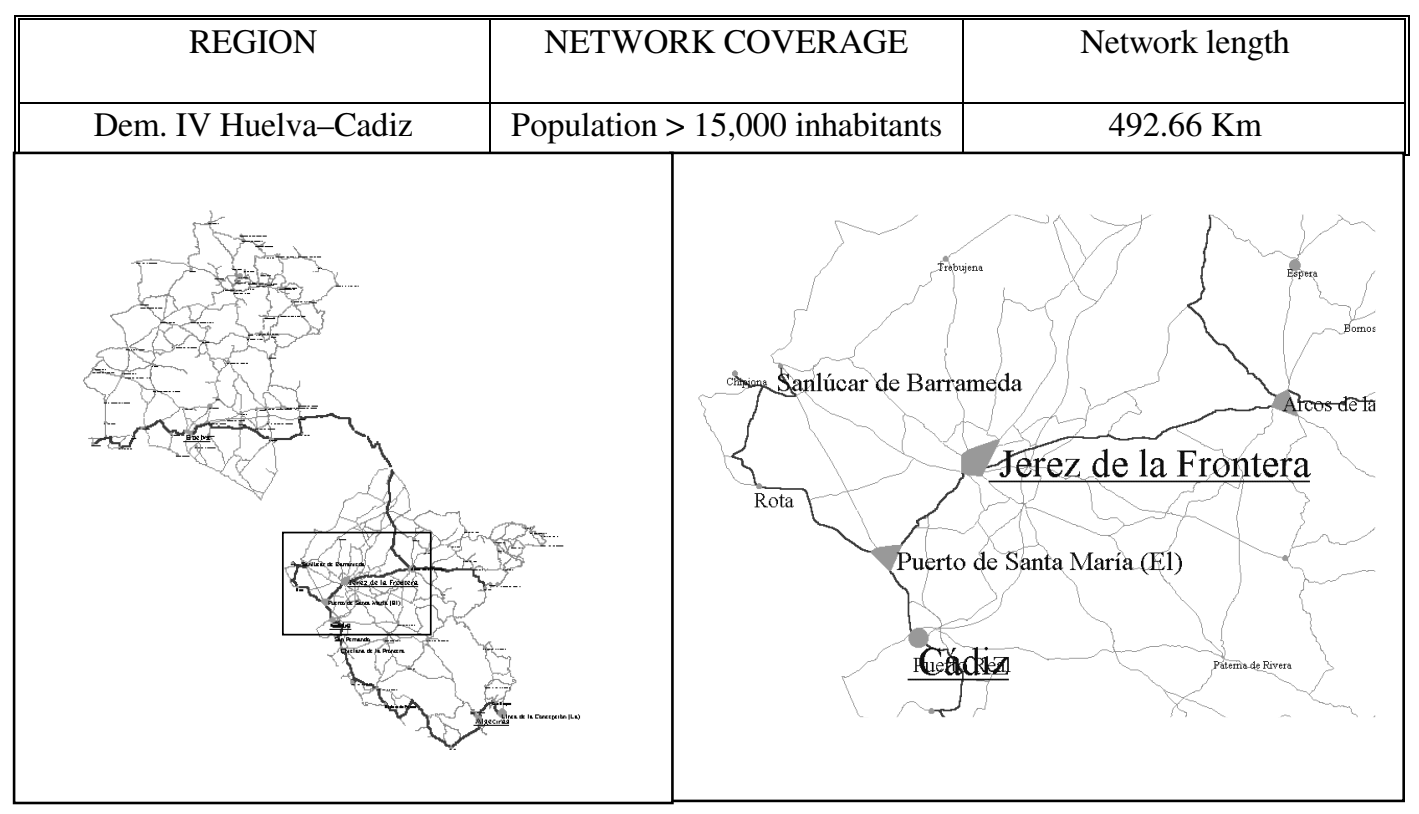

Figure 8 Demarcation IV

Finally, population criteria selecting municipalities larger than 15,000 inhabitants is shown for Demarcation IV. This demarcation offers an additional difficulty because it includes the Doñana Natural Park, one of the largest protected natural reserves in 
Europe, which imposes the constraint that the network connection has to be built across the Seville province. The figure depicts one of the more adequate possibilities to avoid the park. However, firms offering telecommunication services simultaneously in both Demarcations (III and IV) would have to integrate them and considerate the associated new alternatives.

\section{Evolutionary investment scenarios}

Another successful capacity of the tool is the construction of telecommunication networks in several stages. In this context, the DSS lets the user test and analyze evolutionary investment scenarios. This special characteristic allows the firms to consider investment alternatives according to their strategic planning.

Figure 9 reflects an evolutionary scenario over Demarcation III (including Seville and its metropolitan area), in which municipalities are incorporated in successive stages depending on its population size and allowing the company to temporarily schedule its investment efforts. The first stage serves towns larger than 50,000 inhabitants. Thus, after the first stage the network only includes the three largest municipality in the region. The second stage lowers the population requirement to municipalities down to 25,000 inhabitants. As a consequence most of the main towns of the metropolitan area of Seville are connected and served. Finally the third stage lowers the requirement to every other municipalities with population size larger than 10,000 
inhabitants.

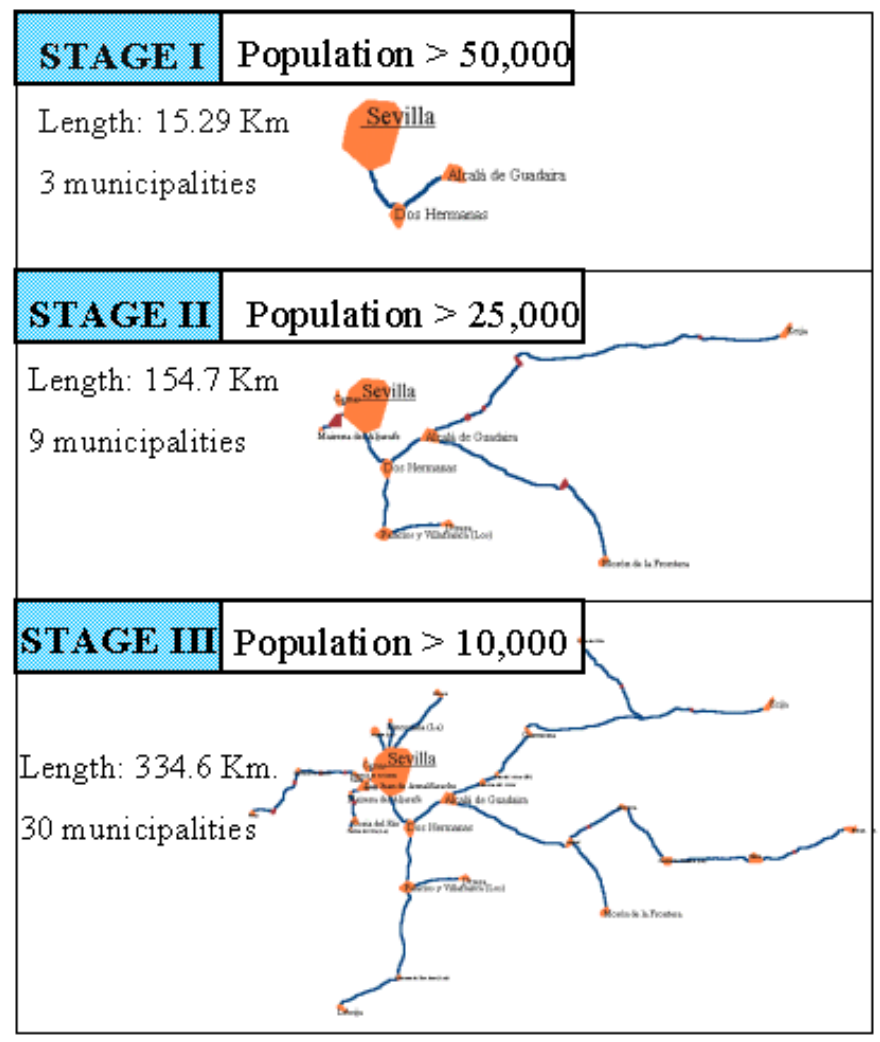

Figure 9 Evolutionary scenarios

\section{Conclusions}

A DSS has been described whose aim is the study of investment decisions associated to the deployment of telecommunication networks with the capacity of analysing diverse scenarios. Economical appraisal is the main consideration in order to value alternatives for most type of projects. This is even more true in the telecommunication sector because of the important investment amounts required for the conduit construction, cable installation, transmitter/receiver/regenerator location 
and hub activation. Investments in cable telecommunications market consider long time horizons, which require in turn multi-stage network development approaches. The DSS proposed is based on the analysis of evolutionary scenarios. These characteristics of the telecommunication network sector make the use of the Decision Support Systems essential. The tool is integrated with a powerful set of mathematical programming models together with a corresponding set of algorithms and an userfriendly graphic interface that allows the interactive analysis of the different scenarios. As a consequence, the tool allows including in the decision process more information and less structured than usually considered. The paper describes the application of the tool to the analysis of the Andalusia region, where cable telecommunication industry is taking the first steps in the development of backbone networks.

The results of this Decision Support System have been validated by the Regional Government of Andalusia that has made use of the tool to establish their proposals to the public auction according to reasonable parameters of investment cost-effectiveness. TEL97-1122), Spain. 


\section{References}

1 Sprague, RH and Carlson, ED (1982). Building Effective Decision Support Systems, Prentice-Hall: Englewood Cliffs, NJ.

2 Wynne, BE (1984). A domination sequence - MS/OR; DSS; and the fifth generation. Interfaces 14: 51-58.

3 Cosares, S, Deutsch, DN, Saniee, I and Wasem, OJ (1995). SONET Toolkit: A Decision Support System for designing robust and cost-effective fiber-optic networks. Interfaces 25: 20-40.

4 Medova, E (1998). High-level languages, mathematical programming solvers \& visualisation tools for B-ISDN Planning. Presented at the INFORMS International Meeting. Tel Aviv, Israel.

5 Onieva, L et al (1996). Determinacion de demarcaciones para la provision de servicios de telecomunicacion por cable en la Comunidad Autónoma Andaluza. Technical Report, Dirección General de Comunicación Social, Consejería de la Presidencia, Junta de Andalucía.

6 Instituto Estadistico de Andalucia (1998). Manual del Sistema de Informacion Municipal de Andalucia. IEA Ed.

7 Connell, NAD and Powell, PL (1990). A comparison of potential applications of Expert Systems and Decision Support Systems. J Opl Res Soc 41: 431-439.

8 Chang, S-G and Gavish, B (1993). Telecommunications network topological design and capacity expansion: Formulations and algorithms. Telecom Sys 1: 
99-131.

9 Tcha, D and Yoon, M (1995). Conduit and cable installation for a centralized network with logical star-star topology. IEEE Trans on Communication 43: 958-967.

10 Yoon, M, Baek, Y and Tcha, D (1998). Design of a distributed fiber transport network with hubbing topology. Eur J Opl Res 104: 510-520.

11 Cortes, P (1999). Multi-injection model to solve the Steiner problem in networks. Technical report IO-03-99T, University of Seville.

12 Lee, K, Park, K and Park, S (1996). Design of capacitated networks with tree configurations. Telecom Sys 6: 1-19.

13 Hall, L (1996). Experience with a cutting plane algorithm for the capacitated spanning tree problem. Informs J on Computing 8: 219-234.

14 Gouveia, L (1993). A comparison of directed formulations for the capacitated minimal spanning tree problem. Telecom Sys 1: 51-76.

15 Winter, P (1987). Steiner problem in networks. Networks 17: 129-167.

16 Aneja, YP (1980). An integer linear programming approach to the Steiner problem in graphs. Networks 10: 167-178. 